\title{
Low-Complexity Signal Detection for Quadrature Spatial Modulation
}

\author{
Yang Jiang ${ }^{1}$, Yahui $\mathrm{Wu}^{2}, \mathrm{Xia} \mathrm{Wu}^{3}, \mathrm{Xia} \mathrm{Chu}^{4}$ and Zonglin $\mathrm{Xie}^{5}$ \\ School of Communication Engineering, Chongqing University, \\ Chongqing, China \\ 1'cqjiangsun@126.com; 2ya_hui_wu@163.com; ${ }^{3} X i a W u @ e q u . e d u . c n$; \\ ${ }^{4}$ chuxia123321@sina.com; ${ }^{5}$ cqzonlym1991@sina.com
}

\begin{abstract}
Quadrature spatial modulation (QSM) is a new digital multiple-input multiple-output (MIMO) transmission technique to further increase the spectral efficiency of spatial modulation (SM). Although the maximum likelihood $(M L)$ detection algorithm is capable of achieving the optimal performance, it necessitates exhaustive search with excessive computational complexity. In this paper, we show that the SD is applicable to QSM. Therefore, an improved SD algorithm for QSM, termed based sorting sphere decoding $(B S-S D)$, is proposed. On one hand, an ordering algorithm is applied to sort the possible transmit antenna combinations (TACs). On the other hand, the possible symbol vectors for each TAC are detected in sequence by using sphere decoding detector algorithm. Simulation results show that SD aided by ordering schemes provides a substantial reduction in the computational complexity while maintaining the optimum bit-error-ratio (BER) performance.
\end{abstract}

Keywords: quadrature spatial modulation (QSM), multiple input multiple output $(M I M O)$, sphere decoding $(S D)$, sorting based sphere decoding (BS-SD)

\section{Introduction}

Multiple-input multiple-output (MIMO) systems stand as an effective way to increase the data rates of today's wireless networks, which offer higher spectral efficiency and reliability by means of diversity and spatial multiplexing gains. Recently proposed schemes reveal that further capacity gains could be achieved by introducing the spatial dimension, coordinated by antenna indices [1]. Spatial modulation (SM) is example of schemes that follow this strategy.SM is a spatial multiplexing MIMO technique that was proposed in [2] to increase the spectral efficiency and overcome inter-channel interference (ICI) [3], which employs only one active transmit antenna in each time slot. Moreover, SM needs only one RF chain, which significantly reduces the hardware complexity of the system [4]. Accordingly, SM appears to be an excellent candidate for massive MIMO [5]-[8]. However, a major criticism of SM is that the data rate enhancement requires a large number of transmit antennas and is proportional to the basetwo logarithm of the number of transmit antennas. Accordingly, enhancing the spectral efficiency of SM techniques has gained significant interest in literature.

Quadrature spatial modulation (QSM) is a method for processing the signal before transmission, in which the spatial constellation symbols are expanded to in-phase and quadrature components[9]. The first dimension transmits the real part of a signal constellation symbol on the cosine carrier and the other one transmits the imaginary part of the constellation symbol on the sine carrier. Hence, an additional bits of base-two logarithm of the number of transmit antennas can be transmitted in QSM, as compared with the conventional SM system. Moreover, QSM system overcomes conventional MIMO drawbacks including inter-channel interference (ICI) since the two transmitted 
data are orthogonal and modulated on the real part and the imaginary part of the carrier signal.

As detection schemes for QSM, a maximum likelihood (ML) detection algorithm was considered in [9] to estimate the active transmit antenna combinations (TACs) and the modulated symbols simultaneously. Although the ML detection algorithm is capable of achieving the optimal system performance, it necessitates exhaustive search with excessive computational complexity. Therefore, it remains an open question to design low-complexity signal detection algorithms for QSM. The sphere decoding (SD) algorithm can avoid an exhaustive search by detecting only those points that lie inside a sphere with radius $C$ [10]. Inspired by scheme of SD algorithm, this paper focuses on developing low-complexity detection algorithm for QSM. Firstly, we show that the SD is able to apply to QSM. We refer to this detection algorithm as QSM-SD. It maintains an almost optimum performance but low complexity. Secondly, to find a lower computational complexity but remain near optimum performance. We propose a novel based sorting sphere decoding (BS-SD) detection algorithm for QSM systems, which is referred as QSM-BS-SD. Firstly, an ordering algorithm is developed to sort the transmit antenna combinations. After that, based on SD method, the possible symbol vectors for each TAC are detected in sequence. Unlike the main idea of QSM-SD, the proposed QSM-BS-SD provides a more efficient computation of the searching points which lie inside a sphere. Numerical results show that the proposed QSM-BS-SD obtains optimal performance, while the required complexity is much lower than those of ML detection algorithm and QSM-SD with large number of transmit antennas and high order modulation.

The remaining parts of this letter are organized as follows. Section II presents QSM system model and the optimal detection technique. In Section III, the QSM-SD and proposed QSM-BS-SD are described, and a theoretical analysis of their computational complexities is performed. Section IV presents the simulation of BER performance comparison and complexity comparison between the proposed QSM-BS-SD detector, QSM-SD and ML detection algorithm. Finally, Section V concludes the letter.

\section{System Model}

\subsection{QSM Modulator}

QSM works as follows [9].The bitstream emitted by a binary source is divided into blocks containing $m=\log _{2}\left(N_{t}^{2} \times M\right)$ bits each at one particular time instant, where $M$ denotes the modulation order of arbitrary M-quadrature amplitude modulation $(M$ $Q A M) /$ phase shift keying or other constellation diagrams. The incoming data bits are processed and partitioned into three groups. A signal constellation symbol is modulated by $\log _{2}(M)$ bits. In addition, two spatial constellation symbols are modulated each by $\log _{2}\left(\mathrm{~N}_{t}\right)$ bits. The signal constellation symbol $x$ is further decomposed to its real ${ }^{\left(x_{\mathfrak{R}}\right)}$ and imaginary $\left(x_{\mathfrak{Y}}\right)$ parts and processed by a single IQ RF chain to yield

$$
s=\operatorname{Re}\left[x e^{-j 2 \pi f c t}\right]=x_{\Re} \cos (2 \pi f c t)+x_{\mathfrak{J}} \sin (2 \pi f c t)
$$

Hence, ${ }^{x_{\mathfrak{R}}}$ is transmitted over the cosine carrier, whereas ${ }_{\mathfrak{\Im}}$ is transmitted over the sine carrier. The QSM system model is depicted in Figure 1 and an example is shown for one time instance using QPSK and four transmit antennas. The incoming data sequence $b=110100$. The first $\log _{2}(M)$ bits [1 1 ] modulate a QPSK symbol $x=+1+i$. The second $\log _{2}\left(N_{t}\right)$ bits [0 1$]$ modulate the active antenna index $l_{\Re}=2$ to 
transmit ${ }_{\Re}$ of $x$, and the last $\log _{2}\left(N_{t}\right)$ bits [0 0 ] modulate the active antenna index $l_{\mathfrak{Y}}=1$ to transmit $x_{\mathfrak{I}}$ of $x$.Then, the transmitted vector is able to be expressed as

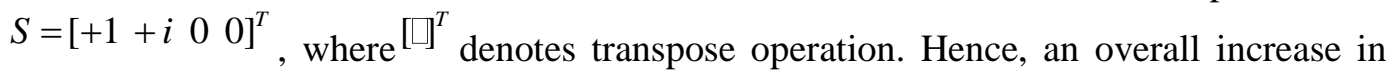
spectral efficiency by $\log _{2}\left(N_{t}\right)$ as compared to SM system is achieved. The mapping table with 2 transmit antennas and QPSK modulation is depicted in Table 1.

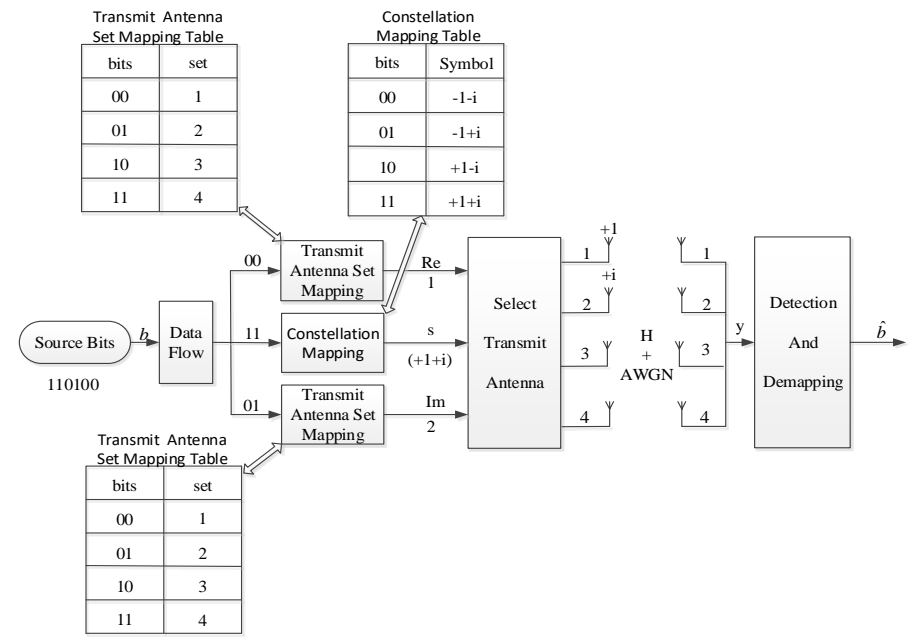

Figure 1. Quadrature Spatial Modulation System Model

Table 1. The Mapping Table of Quadrature Spatial Modulation

\begin{tabular}{lcccccc}
\hline \hline $\begin{array}{l}\text { Transmit } \\
\text { bitstream }\end{array}$ & Space bit & $\begin{array}{l}\text { Real } \\
\text { antenna } \\
\text { index }\end{array}$ & $\begin{array}{c}\text { Imag } \\
\text { antenna } \\
\text { index }\end{array}$ & Modulation bit & $\begin{array}{c}\text { Modulation } \\
\text { symbols }\end{array}$ & $\begin{array}{l}\text { Output } \\
\text { vectors }\end{array}$ \\
\hline 0000 & 00 & 1 & 1 & 00 & $-1-\mathrm{j}$ & {$[-1-\mathrm{j} 0]$} \\
0001 & 00 & 1 & 1 & 01 & $-1+\mathrm{j}$ & {$[-1+\mathrm{j} 0]$} \\
0010 & 00 & 1 & 1 & 10 & $+1-\mathrm{j}$ & {$[+1-\mathrm{j} 0]$} \\
0011 & 00 & 1 & 1 & 11 & $+1+\mathrm{j}$ & {$[+1+\mathrm{j} 0]$} \\
0100 & 01 & 1 & 2 & 00 & $-1-\mathrm{j}$ & {$[-1-\mathrm{j}]$} \\
0101 & 01 & 1 & 2 & 01 & $-1+\mathrm{j}$ & {$[-1+\mathrm{j}]$} \\
0110 & 01 & 1 & 2 & 10 & $+1-\mathrm{j}$ & {$[+1-\mathrm{j}]$} \\
0111 & 01 & 1 & 2 & 11 & $+1+\mathrm{j}$ & {$[+1+\mathrm{j}]$} \\
1000 & 10 & 2 & 1 & 00 & $-1-\mathrm{j}$ & {$[-\mathrm{j}-1]$} \\
1001 & 10 & 2 & 1 & 01 & $-1+\mathrm{j}$ & {$[+\mathrm{j}-1]$} \\
1010 & 10 & 2 & 1 & 10 & $+1-\mathrm{j}$ & {$[-\mathrm{j}+1]$} \\
1011 & 10 & 2 & 1 & 11 & $+1+\mathrm{j}$ & {$[+\mathrm{j}+1]$} \\
1100 & 11 & 2 & 2 & 00 & $-1-\mathrm{j}$ & {$[0-1-\mathrm{j}]$} \\
1101 & 11 & 2 & 2 & 01 & $-1+\mathrm{j}$ & {$[0-1+\mathrm{j}]$} \\
1110 & 11 & 2 & 2 & 10 & $+1-\mathrm{j}$ & {$[0+1-\mathrm{j}]$} \\
1111 & 11 & 2 & 2 & 11 & $+1+\mathrm{j}$ & {$[0+1+\mathrm{j}]$} \\
\hline \hline
\end{tabular}




\subsection{Channel Model}

A generic $N_{r} \times N_{t}$ MIMO configuration is considered with $N_{t}$ and $N_{\mathrm{r}}$ being the number of transmit and receive antennas, respectively. The modulated vector $s$ is transmitted through a frequency-flat Rayleigh fading channel with transfer function $\mathrm{H}$. Thus, the entries of $\mathrm{H}$ are modeled as complex independent and identically distributed (i.i.d.) Gaussian random variables with zero mean and unit variance. Moreover, a perfect channel state information (CSI) at the receiver is assumed, but with no CSI at the transmitter. Thus, the $N_{r} \times 1$ received vector can be written as follows:

$$
\begin{aligned}
y & =H S+n \\
& =h_{l_{\mathfrak{R}}} x_{\mathfrak{R}}+j h_{l_{\mathfrak{\Im}}} x_{\mathfrak{J}}+n, \quad l_{\mathfrak{R}}, l_{\mathfrak{I}}=1,2, \ldots, N_{t},
\end{aligned}
$$

where $n$ is the $N_{r}$ dimensional Additive White Gaussian Noise (AWGN) with zero mean and variance $\sigma^{2}$ per dimension at the receiver input, $h_{l_{\Re}}$ and $h_{l_{\mathfrak{s}}}$ are the $l_{\Re}-t h$ and $l_{\mathfrak{I}}-$ th column of $\mathrm{H}$, respectively.

\subsection{Detection Algorithm}

In QSM system, assuming perfect channel state information (CSI) at the receiver, the optimum ML detection algorithm is given by

$$
\left[\hat{l}_{\mathfrak{R}}, \hat{l}_{\mathfrak{I}}, \hat{x}_{\mathfrak{R}}, \hat{x}_{\mathfrak{I}}\right]=\arg \min _{l_{\mathfrak{R}}, l_{\mathfrak{F}}, x_{\mathfrak{R}}, x_{\mathfrak{S}}}\|y-H S\|^{2}
$$

According to (2), (3) can be simplified as follows:

$$
\begin{aligned}
& {\left[\hat{l}_{\mathfrak{R}}, \hat{l}_{\mathfrak{I}}, \hat{x}_{\mathfrak{R}}, \hat{x}_{\mathfrak{I}}\right]=\arg \min _{l_{\Re}, l_{\mathfrak{\Im}}, x_{\mathfrak{R}}, x_{\mathfrak{I}}}\left\|y-\left(h_{l_{\mathfrak{R}}} x_{\mathfrak{R}}+j h_{l_{\mathfrak{\Im}}} x_{\mathfrak{I}}\right)\right\|^{2}} \\
& =\arg \min _{l_{\Re}, l_{\mathfrak{\Im}}, x_{\mathfrak{R}}, x_{\mathfrak{J}}}\left\{\sum_{r=1}^{N_{r}}\left|y_{r}-\left(h_{l_{\mathfrak{\Re}}, r} x_{\mathfrak{R}}+j h_{l_{\mathfrak{S}}, r} x_{\mathfrak{J}}\right)\right|^{2}\right\},
\end{aligned}
$$

where $\bullet$ identifies the estimated antenna labels and constellation symbols, $\|\cdot\|_{\text {denotes the }}$ norm. The detected antenna indexes $\hat{l}_{\mathfrak{R}}$ and $\hat{l}_{\mathfrak{I}}$ along with the detected data symbols $\hat{x}_{\Re}$ and $\hat{x}_{\mathfrak{I}}$ are used to retrieve the original information bits, where $y_{r}$, $h_{l_{\Re}, r}$ and $h_{l_{\Im}, r}$ are the $r-t h$ entries of $y, h_{l_{\Re}}$ and $h_{l_{\mathfrak{s}}}$, respectively.

\section{New Low-complexity Sphere Decoders for QSM}

In this section, we introduce two SDs modified algorithm for QSM. The first one (QSM-SD) aims at reducing the number of summations over receive antennas in (4). The second one (QSM-BS-SD) combines an ordering algorithm and SD decoders in order to provide a more efficient computation of the searching points which lie inside a sphere.

\subsection{QSM-SD Detection Algorithm}

The QSM-SD detection algorithm considered in this paper is a modified version of the $\mathrm{SD}$ algorithm presented in [11], by adopting the tree search structure, as shown in Figure 2.The QSM-SD performs a ML search only on paths that lead to points $\left(\hat{l}_{\mathfrak{R}}, \hat{l}_{\Im}, \hat{x}_{\Re}, \hat{x}_{\Im}\right)$ with 
an error and are less than or equal to the sphere radius $C$. In this paper, the initial radius of the QSM-SD detection algorithm is adjusted according to the noise level assuming that the knowledge of the SNR at the receiver side is known from previous received data. Then the radius is adapted when a point is found inside the sphere by the Euclidean distance of that point. The initial sphere radius considered in QSM-SD is a function of the noise variance as given in [12]

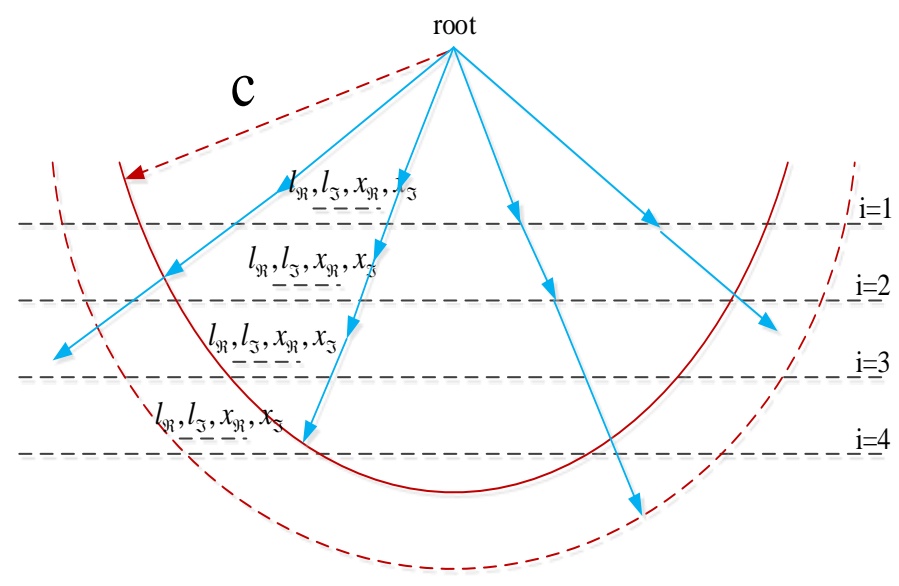

Figure 2. The Tree Structure and Sphere Constraint for the Sphere Decoder

$$
C^{2}=2 \alpha N_{r} \sigma_{n}^{2},
$$

where $\sigma_{n}^{2}$ is the noise variance and $\alpha$ is a constant chosen to maximize the probability of having the transmitted point inside the sphere. Depending on the SNR value, a major reduction in the number of calculated paths can be achieved. The detector can formally be written as follows:

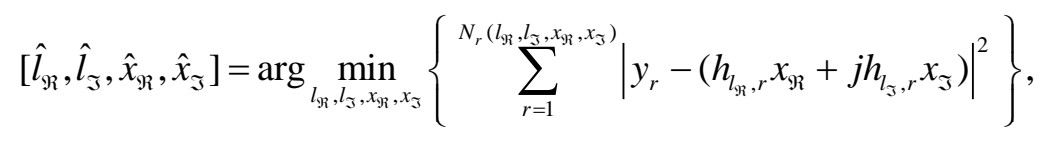

where

$$
N_{r}\left(l_{\Re}, l_{\Im}, x_{\Re}, x_{\Im}\right)=\underset{n \in\left\{1,2, \cdots N_{r}\right\}}{\arg \max }\left\{n\left|\sum_{r=1}^{n}\right| y_{r}-\left.\left(h_{l_{\Re}, r} x_{\Re}+j h_{l_{\Im}, r} x_{\Im}\right)\right|^{2}<C^{2}\right\}
$$

QSM-SD detection algorithm searches the paths leading to each point $(l, x)$ as long as it is still inside the sphere as depicted in Figure 2. Whenever a point is found to be inside the sphere, the radius $C$ is updated with the Euclidean distance of that point. The path with the minimum Euclidean distance is considered to be the solution. QSM-SD detection algorithm selects the path with the minimum Euclidean distance even if all the points were outside the sphere. We regard the point with the minimum Euclidean distance and $N_{r}(\cdot,,, \cdot, \cdot)=N_{r}$ as the optimal solution.

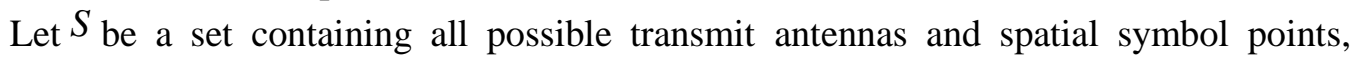
$S=\left\{(l, x): l \in\left[1, \ldots, N_{t}\right], x \in\{M\}\right\}, \delta$ be the Euclidean distance error, and $\varphi_{\text {be the depth }}$ 
of the search on each path ${ }^{(l, x)}$.Then, the key procedure for QSM-SD can be explained as Table 2.

\section{Table 2. The Key Procedure for QSM-SD}

Input: Channel matrix $H$, receive vector ${ }^{y}$, initial sphere radius $C$, transmitting antenna sets $l$ and constellation symbol sets $x$.

Output: Estimated antenna vector $\hat{l}_{\mathfrak{R}}, \hat{l}_{\Im \text { and constellation symbol }} \hat{x}_{\mathfrak{R}}, \hat{x}_{\mathfrak{\Im}}$.

(1) For: $l_{\Re}, l_{\mathfrak{I}} \in l ; x_{\Re}, x_{\mathfrak{I}} \in x ;(l, x) \in S$

(a) For: $i=1: N_{r}$

(i) $\delta\left(l_{\mathfrak{R}}, l_{\mathfrak{Y}}, x_{\mathfrak{R}}, x_{\mathfrak{Y}}\right)+=\left|\mathrm{y}_{i}-\left(h_{l_{\mathfrak{Y}}, \mathrm{i}} x_{\mathfrak{R}}+j h_{l_{\mathfrak{Y}}, \mathrm{i}} x_{\mathfrak{Y}}\right)\right|^{2}$

(ii) if $\delta\left(l_{\mathfrak{R}}, l_{\Im}, x_{\mathfrak{r}}, x_{\Im}\right) \geq C^{2}$, then go to 1

(iii) $\varphi\left(l_{\Re}, l_{\Im}, x_{\mathfrak{R}}, x_{\Im}\right)+=1$

(b)

$$
\begin{aligned}
& S_{\varphi}\left(l_{\mathfrak{R}}, l_{\mathfrak{I}}, x_{\mathfrak{R}}, x_{\mathfrak{I}}\right)=\underset{l_{\mathfrak{H}}, l_{\mathfrak{S}} \in l, x_{\mathfrak{\Re}}, x_{\mathfrak{Y}} \in x}{\arg }\left(\varphi\left(l_{\mathfrak{R}}, l_{\mathfrak{I}}, x_{\mathfrak{R}}, x_{\mathfrak{I}}\right)=\max (\varphi)\right) \\
& \text { (3) }
\end{aligned}
$$

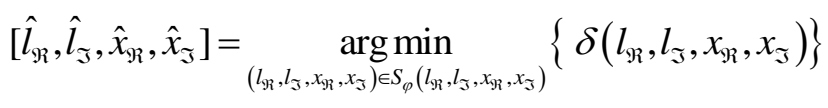

\subsection{Proposed QSM-BS-SD Detection Algorithm}

In the proposed QSM-BS-SD detection algorithm, the first stage we sort the possible TACs[13] by an ordering algorithm. Further to say, we compute the pseudo-inverse of each channel column to preprocess the received vector $\mathrm{y}$, which is as follows

$$
p_{i}=\left(h_{i}\right)^{\dagger} y
$$

where

$$
\left(h_{i}\right)^{\dagger}=\frac{h_{i}^{H}}{h_{i}^{H} h_{i}} \text { and } i \in\left\{1,2, \cdots, \mathrm{N}_{t}\right\} .
$$

After obtained $P=\left[\mathrm{p}_{1}, p_{2}, \cdots, p_{N_{T}}\right]^{T}$, to examine the dependability of each TAC, a weighting coefficient $w_{k}$ is introduced to as

$$
\omega_{k}=p_{k_{\mathfrak{l}}}^{2}+p_{k_{l \mathfrak{F}}}^{2},
$$

where $^{k \in\{1,2, \cdots N\},} N=N_{t}+\left(\begin{array}{c}N_{t} \\ 2\end{array}\right)$, and $l_{\mathfrak{R}}, l_{\mathfrak{I}} \in\left\{1,2, \cdots N_{T}\right\}$.

After obtaining $W=\left[w_{1}, w_{2}, \cdots w_{N}\right]^{T}$, we then sort the weighting coefficient values and obtain the ordered TACs as

$$
\left[k_{1}, k_{2}, \cdots k_{N}\right]=\arg \operatorname{sort}(\mathrm{W}),
$$


where ${ }^{\operatorname{sort}()}$ defines an ordering function for reordering the elements of the input vector in descending order, and $k_{1}, k_{N}$ are the indices of the maximum and minimum value in $\mathrm{W}$, respectively. In this case, we obtain the ordered TACs with the weighting indices $\left[k_{1}, k_{2}, \cdots k_{N}\right]$. Then, for the obtained ordered TACs, the possible TAC vector is detected by SD processing method sequentially [14]. In particular, for each possible TAC, SD is used to detect symbol $\left[\hat{x}_{j_{\mathfrak{R}}}, \hat{x}_{j_{\mathfrak{Y}}}\right]$ and antenna $\left[\hat{l}_{j_{\mathfrak{R}}}, \hat{l}_{j_{\mathfrak{s}}}\right]$ from the $j$-th TAC, which can be written as follows:

$$
\begin{aligned}
& {\left[\hat{l}_{j_{\mathfrak{R}}}, \hat{l}_{j_{\mathfrak{\Im}}}, \hat{x}_{j_{\mathfrak{H}}}, \hat{x}_{j_{\mathfrak{\Im}}}\right]=\arg \min _{j} \sum_{r=1}^{N_{r}\left(l_{l_{\Re}}, l_{\mathfrak{S}}, x_{\Re}, x_{\mathfrak{\Im}}\right)}\left|y_{r}-h_{k_{j}, r} x\right|^{2}}
\end{aligned}
$$

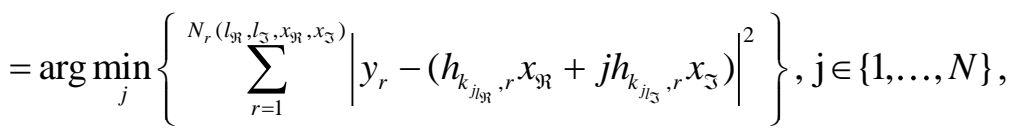

where

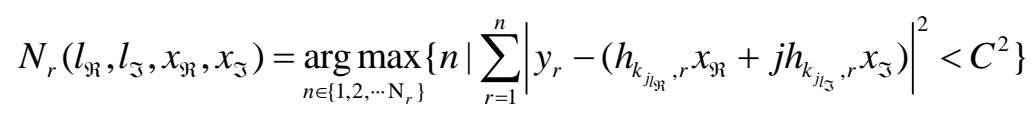

Like QSM-SD, we also regard the point with the minimum Euclidean distance and $N_{r}(\cdot, \cdot, \cdot)=,N_{r}$ as the optimal solution. Then, the key procedure for QSM-SD can be explained as Table 3 .

\subsection{Analysis of Computational Complexity}

In this subsection, we analyze the complexities of the proposed detectors, which are defined as the total number of real multiplication and division operations needed by each algorithm. In addition, we give the computational complexities of ML detection algorithm, QSM-SD, and the proposed QSM-BS-SD as follows.

1) The computational complexity of ML detection algorithm

According to Section IV in [9], the computational complexity of ML is

$$
C_{M L}=8 N_{r} 2^{m}
$$

Note that 8 real multiplications are required to evaluate the Euclidean distance $\left\|y-\left(h_{l_{\mathfrak{R}}} x_{\Re}+j h_{l_{\mathfrak{S}}} x_{\mathfrak{I}}\right)\right\|^{2}$.It needs to be computed $N_{r} 2^{m}$ times, which is equivalent to the complexity of SM [2].

2) The computational complexity of QSM-SD

The computational complexity of the QSM-SD receiver is given by [10]:

$$
C_{S D}=8 \sum_{l=1}^{N_{t}} \sum_{l=1}^{N_{t}} \sum_{x_{\Re=1}}^{\sqrt{M}} \sum_{x_{\mathfrak{\Im}}=1}^{\sqrt{M}} N_{r}\left(l_{\mathfrak{R}}, l_{\mathfrak{\Im}}, x_{\Re \mathfrak{R}}, x_{\mathfrak{\Im}}\right)
$$

Therefore, $C_{S D}$ lies in the interval $8 \times 2^{m} \leq C_{S D} \leq 8 \times 2^{m} N_{r}$, where the lower and upper bound are achieved when $N_{r}\left(l_{\mathfrak{R}}, l_{\mathfrak{\Im}}, x_{\mathfrak{R}}, x_{\mathfrak{\Im}}\right)=1_{\text {and }} N_{r}\left(l_{\mathfrak{R}}, l_{\mathfrak{I}}, x_{\mathfrak{R}}, x_{\mathfrak{\Im}}\right)=\mathrm{N}_{r}$, respectively.

3) The computational complexities of the proposed QSM-BS-SD

The proposed QSM-BS-SD can be described in Table 3. The following factors are taken into account to evaluate the computational complexities of the proposed QSM-BSSD. 


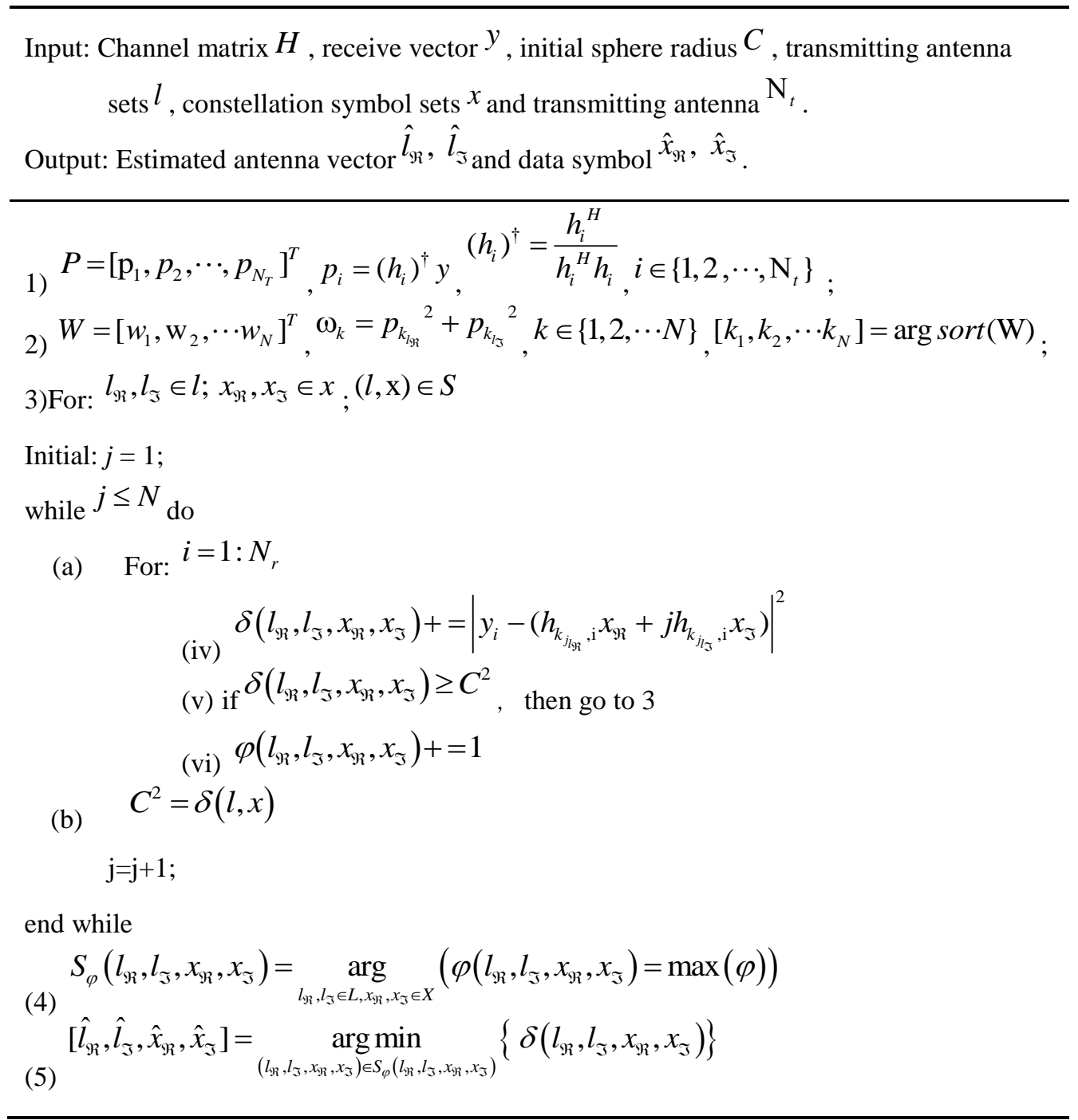

a) The computation of $p_{i}=\left(h_{i}\right)^{\dagger} y$ needs $4 N_{r}+2 N_{r}+4 N_{r}$ real multiplications, where $i \in\left\{1,2, \cdots, \mathrm{N}_{t}\right\}$.

b) Calculating $\quad \omega_{k}=p_{k_{l_{\mathfrak{R}}}}{ }^{2}+p_{k_{l_{\mathfrak{S}}}}{ }^{2} \quad$,where $\quad k \in\{1,2, \cdots N\} \quad$ and $l_{\mathfrak{R}}, l_{\mathfrak{I}} \in\left\{1,2, \cdots N_{T}\right\}$.Evaluating $p_{k_{\mathfrak{\Re}}}{ }^{2}$ and $p_{k_{l_{\mathfrak{F}}}}{ }^{2}$ has same computational complexities, which need 4 real multiplications.

c) The complexity of SD, which is the same as QSM-SD.

The computational complexity of QSM-BS-SD detector is counted as

$$
\begin{aligned}
C_{B S-S D} & =\left(4 N_{r}+2 N_{r}+4 N_{r}\right) N_{t}+4 N_{t}+C_{S D} \\
& =10 N_{r} N_{t}+4 \mathrm{~N}_{t}+C_{S D}
\end{aligned}
$$

where $C_{S D}$ indicates the computational complexity of $\mathrm{SD}$ processing method, which is similar to QSM-SD. 


\section{Simulation Results and Analysis}

In this section, Monte Carlo simulation results for Rayleigh fading channel [15]at least $10^{6}$ channel realisations are considered to compare the performance of the proposed QSM-BS-SD and QSM-SD with the ML. Moreover, the computational complexities of the proposed QSM-BS-SD detector and the QSM-SD are also presented to compare with that of the ML.

\subsection{Performance Comparing in SM and QSM}

The BER performance versus signal to noise ratio (SNR) for $N_{t}=4, N_{r}=4$ and $m=6$ is depicted in Figure 3, where QSM and SM are modulated using QPSK and 16QAM modulation, respectively. From Figure 3, it can be obviously observed that both SD and BS-SD in SM and QSM offer a near optimum performance and the results overlap with ML detection algorithm, respectively. Furthermore, obtained results also demonstrate the significant enhancements of the QSM system over SM system, where a gain of about 3-4 $\mathrm{dB}$ can be achieved. It is important to note that these enhancements are achieved at almost no cost. From (13), the receiver complexity of the QSM scheme and that of SM are equivalent and depend on the considered spectral efficiency.

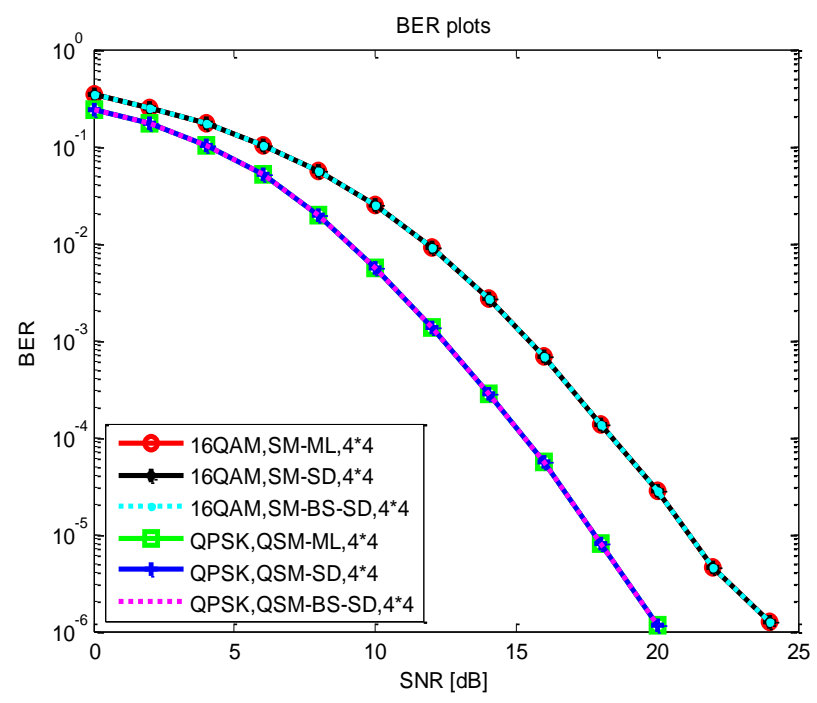

Figure 3. BER Performance Versus $\mathrm{SNR}$ for $\mathrm{Nr}=4, \mathrm{Nt}=4$ and $\mathrm{m}=6$

\subsection{Performance Analysis}

The BER results versus SNR for the two different modulations (QPSK and 16QAM) for $N_{r}=4$ and $N_{t}=4$ are depicted in Figure 4. An important observation is that both modulation methods by using QSM-SD and QSM-BS-SD have the same BER performance as ML detection algorithm. Furthermore, we notice that by using QPSK modulation a better BER performance is achieved compared to the case using 16QAM modulation. As expected, the performance of QSM improves when the modulation order decreases. The BER results versus SNR for the two different transmit antennas $\left(N_{t}=2\right.$ and $N_{t}=8$ ) for $N_{r}=4$ and 8QAM modulation are depicted in Figure 5. It is noticed that for a certain number of transmit antennas the methods using QSM-SD and QSM-BS-SD also have the same performance as ML detection algorithm. Figure 5 shows that when $N_{t}=2$, the BER performance is better than that with $N_{t}=8$. As expected, the performance of QSM improves when transmit antenna decreases. 


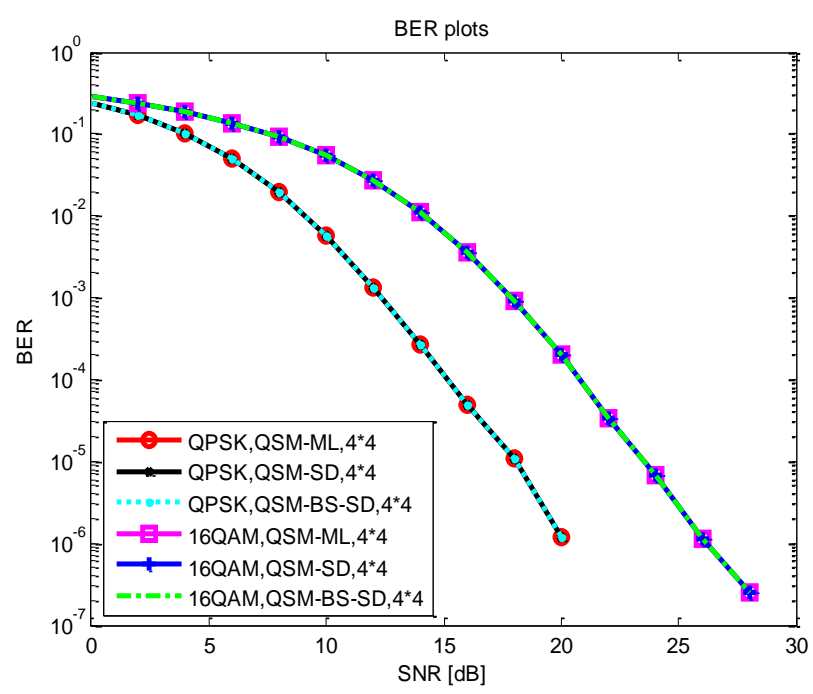

Figure 4. BER Performance Versus $\mathrm{SNR}$ for $\mathrm{Nr}=4$ and $\mathrm{Nt}=4$

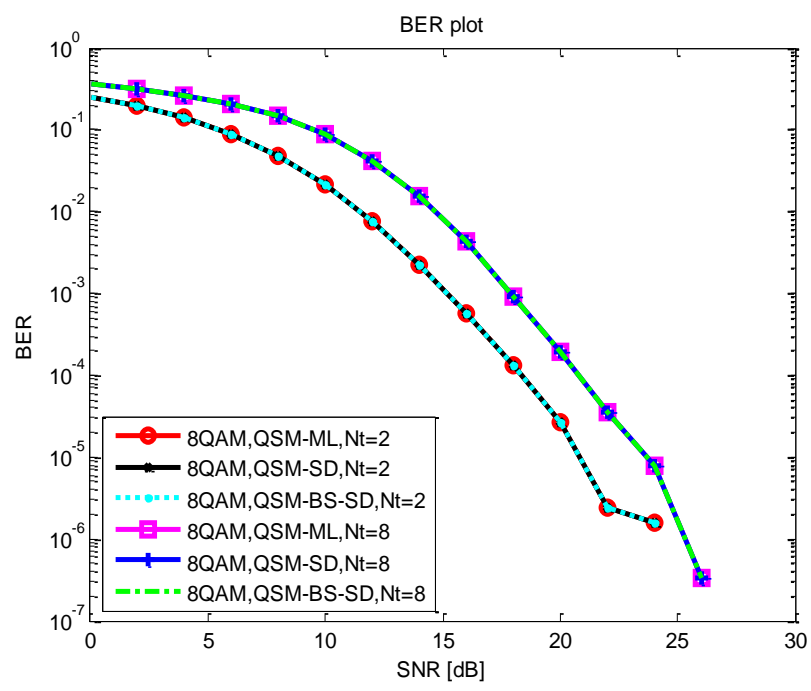

Figure 5. BER Performance Versus SNR for $\mathrm{Nr}=4$

\subsection{Complexity Analysis}

Figures 6 and 7 show the computational complexities of QSM-BS-SD are compared with the computational complexities of QSM-SD. In particular, the figures show relevant complexity reduction of the $\mathrm{SD}$ with respect to the ML optimum detector, i.e., $\mathrm{C}_{r e}(\%)=100 \times\left(c_{M L}-c_{S D}\right) / \mathrm{c}_{M L}[14]$.

In Figures 6 and 7, under different modulation order and transmit antenna, we can see that the proposed QSM-BS-SD schemes and QSM-SD can significantly reduce the computational complexity with respect to the ML detector for QSM, especially in the case of greater SNR. As shown, QSM-BS-SD offers the lowest relative computation complexity, even more than near 56\% reduction for QPSK and 68\% reduction for 16QAM in Figure 6 and near $65 \%$ reduction for $N_{\mathrm{t}}=4$ and $70 \%$ reduction for $N_{\mathrm{t}}=8$ in 8QAM in Figure 7. The larger the constellation size and transmit antenna, the higher reduction in complexity QSM -BS-SD provides than QSM-SD. which is mainly due to complex precomputation including sorting. It is feasible to increase the spectral efficiency 
by enlarge $N_{\mathrm{t}}$ instead of $M$ for a better BER performance. In general, the proposed QSMBS-SD algorithm is more suitable for high spectral efficiency for QSM with large $N_{\mathrm{t}}$ and $M$.Since the proposed detection algorithm is most closely related to conventional SD for MIMO systems, a main contribution of this paper is that it is required to reduce both, the receive search space to arrive at a significant reduction in receiver complexity while maintaining superior bit-error performance.

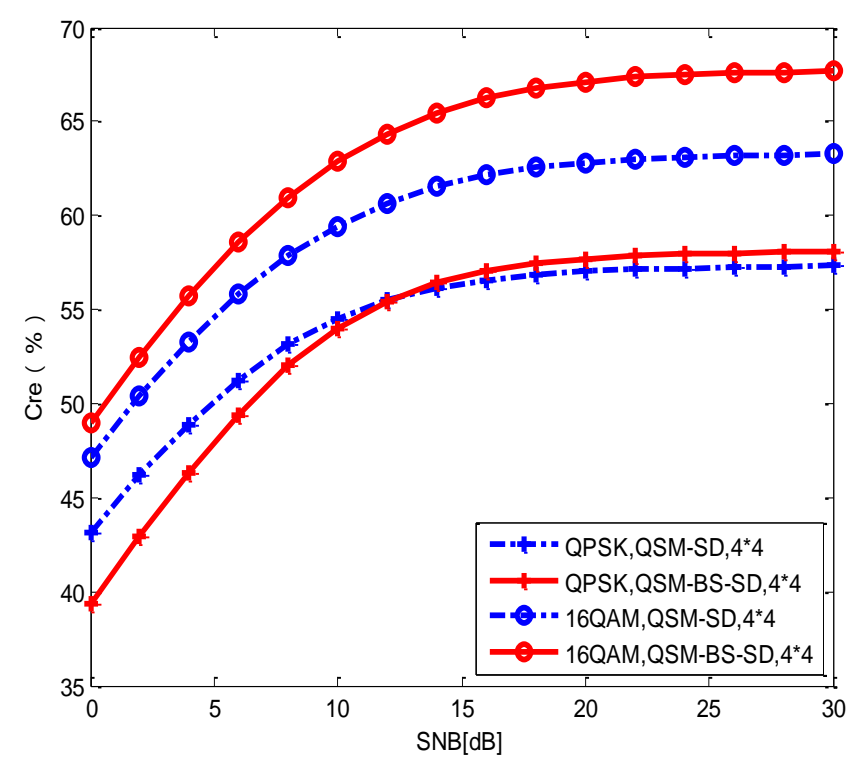

Figure 6. Computational Complexity versus $\mathrm{SNR}$ for $\mathrm{Nr}=4$ and $\mathrm{Nt}=4$

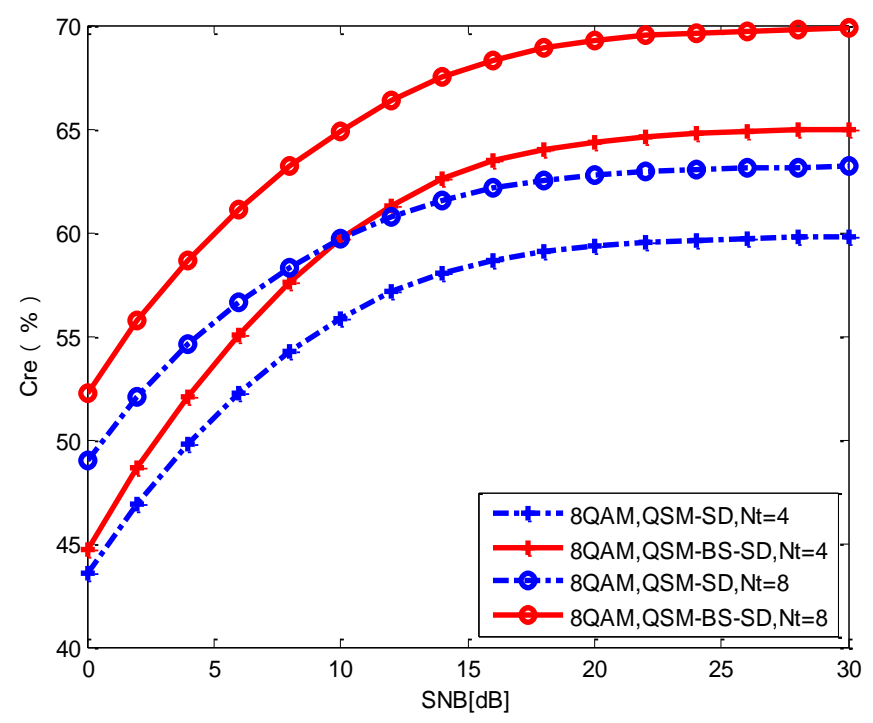

Figure 7. Computational Complexity Versus SNR for $\mathrm{Nr}=4$

\section{Conclusions}

In this paper, we have introduced and analyzed the performance and complexities of proposed QSM-BS-SD and QSM-SD. Simulation results suggest that the proposed QSMBS-SD and QSM-SD have the same performance as the ML detection algorithm. 
Moreover, the proposed QSM-BS-SD and QSM-SD can significantly reduce the computational complexity with respect to the ML detection algorithm, especially the QSM-BS-SD performs closely to its ML detection algorithm counterpart but with a reduction of $70 \%$ in complexity. The BER of BS-SD and SD when used in SM is also compared with the case in QSM. The obtained results demonstrate the significant enhancements of the QSM system over SM system with same spectral efficiency. In general, the proposed QSM-BS-SD is the better solution for high spectral efficiency than QSM-SD and can make an excellent trade-off between performance and complexity by changing the value of modulation order and transmit antenna.

\section{Acknowledgments}

The authors would like to thank the anonymous reviewers for their constructive comments and suggestions.

\section{References}

[1] Y. Yang and B. Jiao, "Information-guided channel-hopping for high data rate wireless communication," IEEE Commun. Lett., vol. 12, no. 4, (2008), pp. 225-227.

[2] R. Mesleh, H. Haas, S. Sinanovic, C.W. Ahn and S. Yun, "Spatial' Modulation", IEEE Transaction Vehicular Technology, vol. 57, no. 4, (2008), pp. 2228-2241.

[3] M.D. Renzo, H. Haas and P.M. Grant, "Spatial modulation for multiple-antenna wireless systems: a survey”, IEEE Communication Magazine, vol. 49, no. 12, (2011), pp. 182-191.

[4] J. Jeganathan, A. Ghrayeb, L. Szczecinski, and A. Ceron, "Space shift keying modulation for MIMO channels," IEEE Trans. Wireless Commun., vol. 8, no. 7, (2009), pp. 3692-3703.

[5] M. Di Renzo and H. Haas, "Bit Error probability of spatial modulation (SM) MIMO over generalized fading channels," IEEE Trans. Veh.Technol., vol. 61, no. 3, (2012), pp. 1124-1144.

[6] M. Di Renzo and H. Haas, "On transmit-diversity for spatial modulation MIMO: impact of spatialconstellation diagram and shaping filters at the transmitter," IEEE Trans. Veh. Technol., vol. 62, no. 6, (2013).

[7] M. Di Renzo, D. D. Leonardis, F. Graziosi, and H. Haas, "Space shift keying (SSK) MIMO with practical channel estimates," IEEE Trans.Commun., vol. 60, no. 4, (2012), pp. 998-1012.

[8] M. Di Renzo and H. Haas, "Bit error probability of space-shift keying MIMO over multiple-access independent fading channels," IEEE Trans.Veh. Technol., vol. 60, no. 8, (2011), pp. 3694-3711.

[9] R. Mesleh, S. S. Ikki, and el Hadi M. Aggoune, "Quadrature Spatial Modulation," IEEE Trans. Veh. Technol., vol. 64, no. 6, (2014), pp. 2738-2742.

[10] A. Younis, R. Mesleh and P. Grant, "Reduced complexity sphere decoder for spatial modulation detection receivers", IEEE GLOBECOM., (2010), pp. 1-5.

[11] A. Younis, S. Sinanovic, M. Di Renzo, R. Y. Mesleh, and H. Haas, "Generalised sphere decoding for spatial modulation," IEEE Trans.Commun., vol. 61, no. 8, (2012), pp. 3656-3664.

[12] B. Hassibi and H. Vikalo, "On the Sphere-Decoding Algorithm I.Expected Complexity," IEEE Trans. on Signal Process., vol. 53, no. 8,(2005),pp. 2806-2818.

[13] Y. Xiao, Z. Yang, L. Dan, P. Yang, L. Yin, and W. Xiang, "Lowcomplexity signal detection for generalized spatial modulation," IEEE Commun. Lett., vol. 18, no. 3, (2014), pp. 403-406.

[14] A. Younis, M.D. Renzo and H. Haas, "Sphere decoding for spatial modulation", IEEE ICC., (2011), pp. 1-6.

[15] M.K. Simon and M.S. Alouini, "Digital Communication over Fading Channels", 1st ed. Hoboken, NJ: Wiley, (2000).

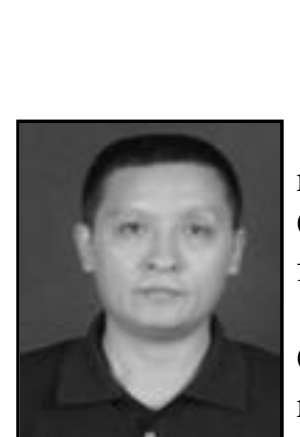

\section{Authors}

Yang Jiang, he was born in Sichuan Province, China, in 1963. He received his first class honors B.S. degree in electronics from Chongqing University in 1987, and his M.S. and Ph.D. degrees both from Chongqing University in 2000 and 2011, respectively. Since 1987, he has been with the Department of Electronic Engineering in Chongqing University, where he is currently a professor. His current research interest is in wireless communications, communication systems and Internet of Things technology. 


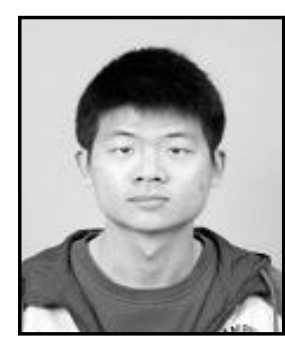

Yahui Wu was born in Henan Province, China, in 1992. He received the B.S. degree from Henan University of Technology, in 2015 in Electronic and Information Engineering. He is currently pursuing the M.S. degree with the College of Communication Engineering, Chongqing University. His research interest is in wireless communications system.

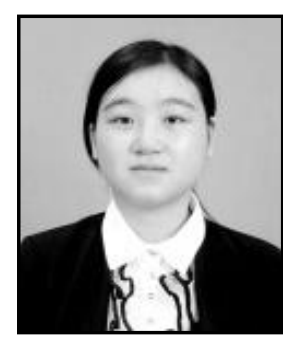

Xia Wu was born in Sichuan Province, China, in 1994. She received the B.S. degree from Shantou University, in 2015 in Electronic Information Engineering. She is currently pursuing the M.S. degree with the College of Communication Engineering, Chongqing University. Her research interest is in wireless communications.

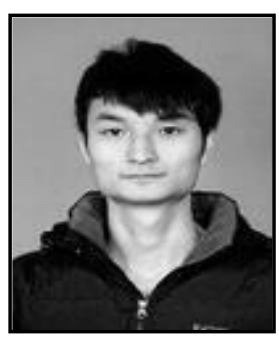

Xia Chu was born in Chongqing Province, China, in 1994. He received the B.S. degree from Chongqing University, in 2015 in Electronic and Information Engineering. He is currently pursuing the M.S. degree with the College of Communication Engineering, Chongqing University. His research interest is in Electronics and communications.

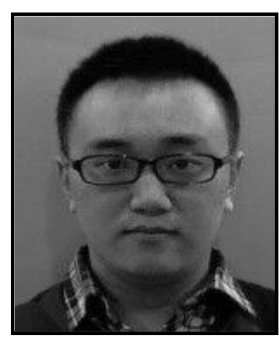

Zonglin Xie was born in Sichuan Province, China, in 1991. He received the B.S. degree from Chongqing University, in 2014 in Communication Engineering. He is currently pursuing the M.S. degree with the College of Communication Engineering, Chongqing University. His research interest is in wireless communications. 
International Journal of Future Generation Communication and Networking Vol.10, No.7 (2017) 